\title{
Effect of Daily Consumption of Lactobacillus reuteri CRL 1098 on Cholesterol Reduction in Hypercholesterolemic Subjects
}

\author{
Agustina Malpeli1, María Pía Taranto², Ricardo C. Cravero3, Marina Tavella1, \\ Victoria Fasano', Dimas Vicentin ${ }^{3}$, Guillermina Ferrari'1, Graciano Magrini3 ${ }^{3}$, Elvira Hébert ${ }^{2}$, \\ Graciela Font de Valdez ${ }^{2}$, Ana M. Varea ${ }^{1}$, Julio M. Tavella ${ }^{1}$, Horacio F. González ${ }^{{ }^{*}}$ \\ ${ }^{1}$ IDIP_Instituto de Desarrollo e Investigaciones Pediátricas "Prof. Dr. Fernando E. Viteri" (Hospital de Niños de \\ La Plata, Ministerio de Salud/Comisión de Investigaciones Científicas de la Provincia de Buenos Aires), La Plata, \\ Argentina \\ ${ }^{2}$ CERELA-Centro de Referencia para Lactobacilos (CONICET), Tucumán, Argentina \\ ${ }^{3}$ Sancor CUL, Departamento de Investigación, Innovación y Desarrollo, Sunchales, Argentina \\ Email: "horaciofgonzalez@gmail.com
}

Received 22 October 2015; accepted 20 December 2015; published 23 December 2015

Copyright (C) 2015 by authors and Scientific Research Publishing Inc.

This work is licensed under the Creative Commons Attribution International License (CC BY).

http://creativecommons.org/licenses/by/4.0/

(c) (i) Open Access

\section{Abstract}

The effect of daily consumption of a yogurt containing Lactobacillus reuteri CRL 1098 on the lipid profile of hypercholesterolemic subjects was evaluated by performing a prospective, randomized, double-blind, cross-over placebo controlled clinical study. Participants consumed daily a yogurt containing $L$. reuteri CRL 1098 or a placebo for four weeks, separated by a wash-out period. Total cholesterol, triacylglycerol, high-density (HDL) and low-density (LDL) lipoprotein levels were assessed at the beginning and at the end of each period. We found a statistically significant reduction of total $(-7.86 \mathrm{~g} / \mathrm{dl})$ and LDL $(-7.02 \mathrm{~g} / \mathrm{dl})$ cholesterol in absolute changes (before-after) as well as a decreasing trend in the group receiving the yogurt containing $L$. reuteri with respect to the placebo group, without detecting changes in HDL-cholesterol and triacylglycerol levels. Our results suggest that low amounts of yogurt (125 g/day) and low doses of the CRL 1098 strain (106 $\mathrm{CFU})$ are sufficient to reduce total and LDL-cholesterol levels in hypercholesterolemic subjects.

\section{Keywords}

Lactobacillus reuteri, Hypercholesterolemia, Cardiovascular Risk, Probiotics, Functional Foods

"Corresponding author.

How to cite this paper: Malpeli, A., et al. (2015) Effect of Daily Consumption of Lactobacillus reuteri CRL 1098 on Cholesterol Reduction in Hypercholesterolemic Subjects. Food and Nutrition Sciences, 6, 1583-1590.

http://dx.doi.org/10.4236/fns.2015.617163 


\section{Introduction}

High serum cholesterol levels are a risk factor for atherosclerosis and cardiovascular disease. In Western countries, cardiovascular disease is one of the leading causes of death, and is extending to Eastern countries to become the main mortality cause worldwide. In Argentina, $46 \%$ of deaths are due to cardiovascular disease, and approximately $30 \%-40 \%$ of the population suffers some kind of hypercholesterolemia [1]-[4].

In the last years, different nutraceutical and functional foods with hypocholesterolemic properties have been developed. In these cases, the effect would be related to the presence of several alternatives such as soy proteins, omega 3, plant sterols, polyphenols in green tea, wine and olive oil, and probiotic microorganisms as selected strains of lactic acid bacteria (LAB) [5] [6].

The use of probiotic LAB strains capable of regulating hyperlipidemia in individuals with hypercholesterolemia is a natural alternative to help maintain healthy lipid levels without undesirable side effects. Research studies show that reduced cholesterol levels due to the use of certain LAB strains could be related to bile salt hydrolase (BSH) activity, present only in microorganisms. $\mathrm{BSH}^{+} \mathrm{LAB}$ strains are able to survive and colonize the small intestine, where the enterohepatic circulation takes place. Once in the intestine, these strains would act on the conjugated bile salts excreted during intake, releasing bile salts or free bile acids which cannot be reabsorbed from the gut and are excreted via the faeces. Under these circumstances, the concentration of bile acids, which should remain constant in the intestine, decreases. To maintain biliary homeostasis, the liver should synthesize more bile acids de novo from endogenous cholesterol. The altered metabolism of bile salts by increased BSH activity would also affect cholesterol metabolism, directly influencing its solubility and subsequent absorption at intestinal level [6]-[8].

Different bifidobacteria and lactobacilli strains (Bifidobacterium lactis, B. longun, Lactobacillus plantarum, $L$. acidophilus, L. reuteri, L. fermentun) would be potentially effective for cholesterol reduction as evidenced through in vitro assays [7]. However, animal and human trials designed to demonstrate serum cholesterol reduction by LAB administration have reported controversial results [7]-[9]. In humans, some trials about the effect of LAB administration have shown decreased serum cholesterol levels [10]-[12], whereas other studies have found no effect [13]-[15].

The assessment of the effect of Lactobacillus (L.) reuteri CRL 1098 on lipid profile has had promising results. Taranto et al. [16] administered a fat-rich diet to mice for 7 days and then the animals were treated with a solution containing $L$. reuteri CRL $1098\left(10^{4}\right.$ cells/day). In the intervention group, total cholesterol and triacylglycerol levels decreased by 38 and $40 \%$, respectively, whereas the HDL/LDL-cholesterol ratio increased by $20 \%$, without producing side effects.

Another report in mice [17] showed that L. reuteri CRL 1098 administration was effective in preventing hypercholesterolemia and further confirmed its permanence in the gut. However, there are no studies reporting the probiotic effect on hypercholesterolemic subjects thus far.

This study was undertaken to evaluate the effect of the daily consumption of L. reuteri CRL 1098-containing yogurt during four weeks on total, HDL-, LDL-cholesterol and triacylglycerol levels in hypercholesterolemic subjects.

\section{Material and Methods}

\subsection{Study Sample and Sample Size Estimation Electing a Template}

Participants were recruited through announcements placed in public institutions from the city of La Plata, Buenos Aires, Argentina. Eligible participants were healthy men and women over the age of 18 years, with hypercholesterolemia (total cholesterol $>200 \mathrm{mg} / \mathrm{dl}$ ) confirmed with laboratory test at the beginning of the study, and not taking any hypocholesterolemic drug.

Inclusion and exclusion criteria of the study sample are summarized in Table 1.

Sample size was estimated to have 90\% power and 95\% confidence interval to detect differences of $20 \mathrm{mg} / \mathrm{dl}$ in cholesterol levels between the intervention and the placebo group at the end of the 4-week study period. Thus, the required sample size was 60 participants in each group ( $20 \%$ loss adjustment; $30 \mathrm{mg} / \mathrm{dl}$ standard deviation).

\subsection{Study Design}

We performed a prospective, randomized, double-blind, cross-over placebo controlled clinical study. Random 
Table 1. Inclusion and exclusion criteria of the study sample.

\begin{tabular}{|c|c|}
\hline Inclusion criteria & Exclusion criteria \\
\hline $\begin{array}{l}\text { Older than } 18 \text { years of age } \\
\text { Total cholesterol levels } \\
\text { higher than } 200 \mathrm{mg} / \mathrm{dl}\end{array}$ & $\begin{array}{l}\text { Use of statins or other cholesterol-lowering drugs within the previous } 6 \text { months. } \\
\text { Use of cholesterol lowering supplements within the previous } 3 \text { months. } \\
\text { Use of systemic antibodies, corticosteroids, androgens or phenitoin. } \\
\text { Lactose intolerance or milk allergy. } \\
\text { History of inflammatory bowel disease or pancreatitis. } \\
\text { Gastrointestinal, renal, hepatic, lung or bile disease. } \\
\text { Hypo/hyperthyroidism. } \\
\text { Menopause. } \\
\text { Family history of hypercholesterolemia. } \\
\text { Chronic use of probiotics ( }>2 \text { doses/week), fiber-based laxatives ( }>2 \text { doses/week) or stimulant laxatives. } \\
\text { Diabetes mellitus or cancer. } \\
\text { Alcohol abuse ( }>40 \text { g/day). } \\
\text { Pregnancy or breastfeeding. }\end{array}$ \\
\hline
\end{tabular}

allocation of study participants to the intervention or placebo group was performed with EPIDAT 3.1 (1:1 allocation ratio).

The study was organized in four visits formed by three consecutive stages that lasted four weeks each. Initially, participants were assigned to consume one yogurt daily (125 g) with (intervention group) and without (placebo group) L. reuteri CRL 1098 during four weeks.

The lipid profile was determined on the first visit; participants were given information and advice on healthy diet and were asked to maintain their habitual lifestyle and to eat the yogurt any time of the day. On the second visit, the lipid profile was determined again and participants began the wash-out period during which no product was consumed. On the third visit, the lipid profile test was repeated and participants of both groups (placebo and intervention) were crossed over for four weeks, i.e., those who had consumed the 125-g placebo yogurt started to eat the intervention yogurt (with L. reuteri CRL 1098), and participants who had received the probiotic-containing yogurt now received the placebo yogurt.

At the end of this stage, on the fourth visit, participants performed the final laboratory test.

On visits 1 and 4, anthropometric parameters (weight, height, waist and abdominal circumference), dietary changes, physical activity practice as well as tobacco and alcohol consumption were recorded together with any side effect.

\subsection{Characteristics of the Intervention Yogurt}

The strain used in this study (L. reuteri CRL 1098) was obtained from the CERELA culture collection (San Miguel de Tucumán, Argentina). The microorganism was cultured in MRS agar at $37^{\circ} \mathrm{C}$ for $16 \mathrm{~h}$. Cells were collected by centrifugation at $6000 / \mathrm{g}$ during $10 \mathrm{~min}$, washed three times with sterile saline solution, suspended in $10 \%$ sterile skimmed milk powder and the biomass produced was finally freeze-dried at Biochemical S.A. (Libertad, Buenos Aires, Argentina). One gram of lyophilized product contained $10^{10} \mathrm{CFU}$; the inoculum was used industrially to produce the intervention yogurt, which contained the same amount of product plus $10^{6} \mathrm{CFU}$ of $L$. reuteri CRL 1098 per gram of yogurt.

\subsection{Control, Follow-Up and Yogurt Preservation}

Both yogurts were produced and prepared by SanCor C.U.L. (Córdoba, Argentina) and filled into 125 g plastic pots.

Addition of the probiotic strain CRL 1098 did not modify the fermentation curve, flavor, texture and consistency of the yogurt, which preserved the same organoleptic properties as the placebo yogurt.

Postacidification was not observed in either the control or the intervention yogurt.

L. reuteri CRL 1098 colony counts during the life span of the product were above the minimum amount established to perform clinical trials (>10E $+06 \mathrm{CFU})$.

\subsection{Data Collection}

Weight, height and waist circumference were taken on visits 1 and 4 . Body weight was measured barefoot and 
in light clothing with a Tanita scale (0.1 g precision, Tanita Corporation of America, Inc. Illinois, USA). Height was measured with a SECA stadiometer (0.5 cm precision, SECA, UK) and waist circumference with an inextensible measuring tape (1 mm precision).

Blood pressure was taken on visits 1 and 4 with an OMROM digital blood pressure monitor.

Physical activity was assessed during visit 1 using the IPAQ questionnaire [18]. The level of physical activity was categorised according to the IPAQ's scoring protocol in multiples of the resting metabolic rate (METS) as low, moderate and intense. Dietary habits were determined with the 14-item Mediterranean Diet Assessment Tool [19]. Tobacco use was measured with the STEPwise approach to chronic disease risk factor surveillance recommended by the World Health Organization [20].

Serum cholesterol (total, LDL and HDL) and triacylglycerol levels were determined by enzymatic reactive kits during each of the four visits (Sigma Chemical Co., St. Louis, MO).

\subsection{Data Analysis}

Data were analysed using SPSS version 19 for Windows. Continuous variables are presented as means \pm standard deviation or medians (interquartile range), and qualitative variables are presented as percentages. All variables were examined for normality using the Kolmogórov-Smirnov test. Excepting triacylglycerol, all variables were normal. Data comparison at the beginning of the study was made with Student's t-test for quantitative variables and Chi-square test for categorical variables. Comparison of initial mean cholesterol levels between groups was performed with Student's t-test and Mann-Whitney Wilcoxon test for normal and non- normal (triacylglycerol) variables, respectively. Before-after differences in cholesterol levels within groups were studied with paired Student's t-test for normal distribution variables and Wilcoxon signed rank test for non- normal distribution variables.

Differences in cholesterol levels between groups were compared with two-factor repeated measures analysis of variance (ANOVA) for normal variables and Friedman Test for triaglylglycerol. In all cases, $P$ values $<0.05$ were considered statistically significant.

\subsection{Ethical Considerations}

Current legal provisions implemented by the Joint Health Research Committee, Ministry of Health of the Province of Buenos Aires, Argentina, were considered. The study protocol was reviewed and approved by said Committee and by the corresponding Institutional Ethics Committee. Participants signed informed consent forms, received oral and written information about the study prior to participation, and could voluntarily abandon the project at any moment. The study was conducted in accordance with the Helsinki Declaration and the current Good Clinical Practice principles.

\section{Results}

A total of 105 healthy adult subjects voluntarily agreed to participate in the study. After excluding those who did not meet the inclusion criteria $(n=35), 70$ subjects were randomly assigned to the study groups. The general characteristics of the placebo and intervention groups at the beginning of the study are presented in Table 2. Age, BMI and blood pressure values were similar in both groups. Half of the patients had a sedentary lifestyle, as determined by IPAQ, and about a quarter of them were smokers. Only waist circumference values were higher in the intervention group $(P=0.043)$.

Table 3 shows that total and LDL-cholesterol levels decreased significantly $(P=0.017$ and $P=0.019$, respectively) in participants that consumed the yogurt containing L. reuteri CRL 1098, whereas HDL-cholesterol and triaclyglycerol levels did not change significantly. On the other hand, no changes could be observed in the placebo group in any of the lipid profile parameters studied.

Although changes in the lipid profile of participants before and after consuming the placebo and the intervention yogurts were not statistically significant, a trend towards lower total cholesterol $(P=0.076)$ and LDL-cholesterol $(P=0.08$ ) levels was found in the intervention group (Table 4).

LDL-cholesterol decreased (3.96\%) in the intervention as compared with the control group, in which case an increase could be detected (3.24\%), showing a significant trend $(P=0.059)$.

Adherence to the Mediterranean diet was similar in both groups; $33.3 \%$ and $29.6 \%$ of participants in the 
Table 2. Clinical characteristics of participants in the placebo and intervention groups.

\begin{tabular}{cccc}
\hline & Placebo $(\mathrm{n}=37)$ & Intervention $(\mathrm{n}=33)$ & $P$ value \\
\hline Age (years) (mean \pm SD) & $42.8 \pm 11.6$ & $47.6 \pm 12.4$ & 0.101 \\
BMI $\left(\mathrm{kg} / \mathrm{m}^{2}\right)($ mean \pm SD) & $26.16 \pm 4.09$ & $27.58 \pm 5.48$ & 0.245 \\
Waist circumference (cm) (mean \pm SD) & $87 \pm 13$ & $94 \pm 13$ & 0.043 \\
Systolic blood pressure (mmHg) (mean \pm SD) & $128 \pm 16$ & $127 \pm 18$ & 0.886 \\
Diastolic blood pressure (mmHg) (mean \pm SD) & $74 \pm 11$ & $72 \pm 11$ & 0.429 \\
Sedentary lifestyle (\%) & 49 & 50 & 0.91 \\
Smoking (\%) & 25 & 26 & 0.94
\end{tabular}

Table 3. Changes in total, HDL-, LDL-cholesterol and triacylglycerol levels in the placebo and intervention groups.

\begin{tabular}{ccccc}
\hline & Placebo & $P$ & Intervention & $P$ \\
\hline $\begin{array}{c}\text { Cholesterol } \\
\text { Abs } \Delta\end{array}$ & $-3.39(24.55)$ & $0.302^{\mathrm{a}}$ & $-7.86(25.46)$ & $0.017^{\mathrm{a}}$ \\
HDL-cholesterol Abs $\Delta$ & $-17.02(7.62)$ & $0.097^{\mathrm{a}}$ & $0.35(9.83)$ & $0.779^{\mathrm{a}}$ \\
LDL-cholesterol Abs $\Delta$ & $0.95(24.68)$ & $0.775^{\mathrm{a}}$ & $-7.02(23.03)$ & $0.019^{\mathrm{a}}$ \\
Triacylglycerol Abs $\Delta$ & $-3.5(-28.75 .17 .75)$ & $0.337^{\mathrm{b}}$ & $1(-29.18)$ & $0.619^{\mathrm{b}}$ \\
\hline
\end{tabular}

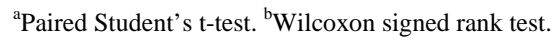

Table 4. Basal data and absolute changes in total, HDL-, LDL-cholesterol and triacylglycerol levels in both study groups.

\begin{tabular}{cccc}
\hline & $\begin{array}{c}\text { Placebo Mean (SD) or Median } \\
\text { (IQR) }\end{array}$ & $\begin{array}{c}\text { Intervention Mean (SD) or Median } \\
(\text { IQR })\end{array}$ & $P$ value \\
\hline Total cholesterol & $224.34(30.72)$ & $230.45(28.76)$ & $0.251^{\mathrm{a}}$ \\
Baseline & $-3.39(24.55)$ & $-7.86(25.46)$ & $0.076^{\mathrm{b}}$ \\
Abs $\Delta^{*}$ & & & $0.340^{\mathrm{a}}$ \\
HDL-cholesterol & $50.32(12.51)$ & $48.23(11.96)$ & $0.828^{\mathrm{b}}$ \\
Baseline & $-17.02(7.62)$ & $0.35(9.83)$ & $0.124^{\mathrm{a}}$ \\
Abs $\Delta^{*}$ & & & $0.088^{\mathrm{b}}$ \\
LDL-cholesterol & $148.59(31.60)$ & $156.97(28.68)$ & \\
Baseline & $0.95(24.68)$ & $-7.02(23.03)$ & $0.957^{\mathrm{c}}$ \\
Abs $\Delta^{*}$ & $106(75.5 .158 .5)$ & $114.5(72.5 .150)$ & $0.680^{\mathrm{d}}$ \\
Triacylglycerol & $-3.5(-28.75 .17 .75)$ & $1(-29.18)$ & \\
Baseline & & & \\
Abs $\Delta^{*}$ & & & \\
\hline
\end{tabular}

${ }^{*}$ Abs $\Delta=$ after-before. ${ }^{\mathrm{a}}$ Student's t-Test. ${ }^{\mathrm{b}}$ Two-factor repeated measures analysis of variance. ${ }^{\mathrm{c}}$ Mann-Whitney Wilcoxon Test. ${ }^{\mathrm{d}}$ Friedman Test.

placebo and intervention groups had scores $\geq 7$ ( $P=0.764)$. During follow-up, no significant changes could be assessed in either group. Smoking and the frequency and intensity of the practice of physical activity remained the same throughout the study period.

\section{Discussion}

In this work, we demonstrate the effect of $L$. reuteri CRL 1098 on hypercholesterolemic subjects through clinical 
trials. Our results show a trend towards lower total and LDL cholesterol levels with the yogurt containing $L$. reuteri CRL 1098. Absolute changes (before-after) with the yogurt containing the probiotic were significant. The decrease in total and LDL-cholesterol was statistically significant ( -7.86 and $-7.02 \mathrm{~g} / \mathrm{dl}$, respectively), whereas HDL-cholesterol and triacylglycerol levels did not change. In the placebo group, the lipid profile did not change significantly.

Several clinical trials tended to demonstrate the effect of probiotic formulations on the lipid profile, obtaining different and controversial results. Most works included microbial BSH+ strains [7] [8] [15]. Administration of 200-ml fermented milk with one strain of Enterococcus faecium and two strains of Streptococcus thermophilus to middle-aged Danish men during 6 weeks reduced LDL-cholesterol by 10\% compared with a placebo yogurt [21]. Two other reports on the administration of milk fermented with the same strains but for a longer period (8 weeks) also showed favourable changes in LDL-cholesterol (adjusted by body weight) [22] and total cholesterol [23] levels compared with the consumption of a placebo. However, when the same strains (E. faecium and $S$. thermophilus) were tested in an English population, lower serum cholesterol levels could not be detected [24], probably due to different dietary habits and intestinal microbiota in the study samples. The effect of a milk product fermented with fructooligosaccharides (FOS) and L. acidophilus LA1 on the lipid profile was also tested [25]. Although the authors reported the reduction of total $(4.4 \% ; P<0.001)$ and LDL-cholesterol $(5.4 \% ; P<$ 0.005), new tests would be necessary to verify whether the lactobacillus or FOS caused such reduction [25]. In another study using the same strain but without FOS, no changes in the lipid profile of participants with normal to borderline high serum cholesterol levels were observed [13]. On the other hand, Anderson \& Gilliland [12] found that administration of milk fermented with L. acidophilus LA1 caused a 3\% reduction in cholesterol levels.

Administration of tablets with L. acidophilus ATCC 4962 and L. bulgaricus ATCC 33409 did not modify serum lipoprotein levels compared with the placebo [26].

Milk fermented with Bifidobacterium longum BL1 decreased total cholesterol only in volunteering subjects with initial cholesterol values $>240 \mu \mathrm{g} / \mathrm{dl}$, showing the potential efficacy of this strain for the treatment of blood lipids [11]. Another report showed that the association of L. acidophilus and B. lactis strains reduced cholesterol levels in hypercholesterolemic subjects compared with a standard placebo yogurt [10]. However, no differences in the lipid profile of women were observed after administration of a yogurt with the same strains included in a conventional yogurt [27].

Jones et al. demonstrated that microencapsulated L. reuteri NCIMB 30242 given in yogurt for six weeks [28] and given as capsules for nine weeks [29] significantly lowered total and LDL-cholesterol levels.

In agreement with the results reported herein, a recent meta-analysis [30] including randomised controlled trials that evaluated the effects of probiotic consumption on blood lipids in 485 participants concluded that probiotics were useful to lower total and LDL-cholesterol levels, but could not modify HDL-cholesterol and triacylglycerol levels.

Our results are positive, since changes in total and LDL-cholesterol could be observed with low amounts of the yogurt (one pot with $125 \mathrm{~g} /$ day) and low concentrations of the strain L. reuteri CRL $1098\left(10^{6} \mathrm{CFU}\right)$. The effective dose of $L$. reuteri CRL 1098 is the lowest reported in the literature for LAB probiotics able to decrease serum lipid without undesirable side effects. Most published works show similar results using higher volumes of the product, in some cases several daily doses, and/or consumption of larger amounts of the probiotic microorganism. Further, L. reuteri CRL1098 can be used as adjunct culture for developing fresh functional foods without requiring strategies to ensure their viability, such as microencapsulation, because this strain is highly resistant to gastrointestinal conditions and adverse technological processes.

\section{Conclusion}

The results obtained in the present study demonstrate that consumption of a 125-g yogurt containing $10^{6} \mathrm{CFU} L$. reuteri CRL 1098 reduced total cholesterol levels by 7.8\% and LDL-cholesterol levels by 7\%, suggesting the clinical importance of such intake. These results are promising, since a review on the primary prevention of myocardial infarction showed that $1 \%$ cholesterol reduction could in turn produce a $6 \%$ to $10 \%$ reduction in the risk for heart disease [31].

\section{Acknowledgements}

This study was funded by FONARSEC (Fondo Argentino Sectorial), Ministerio de Ciencia, Tecnología e Innovación Productiva (id:SGP:02), Argentina. We thank Natalia Alvarez for blood extractions, Andrea Touza for 
product handling and administration, Verónica Molina, Marta Medici, Mariano Obregozo, María José Fornaguera and Lourdez Cruz Pintos for technical assistance with the probiotic strain, and Adriana Di Maggio for correcting the manuscript.

\section{References}

[1] Puska, P., Tuomilehto, J., Nissinen, A., Salonen, J.T., Vartiainen, E., Pietinen, P., Koskela, K. and Korhonen, H.J. (1989) The North Karelia Project: 15 Years of Community-Based Prevention of Coronary Heart Disease. Annals of Medicine, 21, 169-173. http://dx.doi.org/10.3109/07853898909149928

[2] Roy, S. (2014) Atherosclerotic Cardiovascular Disease Risk and Evidence-Based Management of Cholesterol. North American Journal of Medical Science, 6, 191-198. http://dx.doi.org/10.4103/1947-2714.132916

[3] Expert Panel on Detection, Evaluation and Treatment of High Blood Cholesterol in Adults (2001) Executive Summary of the Third Report of the National Cholesterol Education Program (NCEP) (Adult Treatment Panel III). Journal of the American Medical Association, 285, 2486-2497. http://dx.doi.org/10.1001/jama.285.19.2486

[4] World Health Organization (2014) Global Status Report on Noncommunicable Diseases 2014. http://www.who.int/nmh/publications/ncd-status-report-2014/en/

[5] Chen, G., Wang H., Zhang, X. and Tian, Yang S. (2014) Nutraceuticals and Functional Foods in the Management of Hyperlipidemia. Critical Reviews in Food Science and Nutrition, 54, 1180-1201. http://dx.doi.org/10.1080/10408398.2011.629354

[6] Jones, M.L., Tomaro-Duchesneau, C., Martoni, C.J. and Prakash, S. (2013) Cholesterol Lowering with Bile Salt Hydrolase-Active Probiotic Bacteria, Mechanism of Action, Clinical Evidence, and Future Direction for Heart Health Applications. Expert Opinion on Biological Therapy, 13, 631-642. http://dx.doi.org/10.1517/14712598.2013.758706

[7] Kumar, M., Nagpal, R., Kumar, R., Hemalatha, R., Verma, V., Kumar, A., Chakraborty, C.H., Singh, B., Marotta, F., Jain, S. and Yadav, H. (2012) Cholesterol-Lowering Probiotics as Potential Biotherapeutics for Metabolic Diseases. Experimental Diabetes Research, 2012, 2-16. http://dx.doi.org/10.1155/2012/902917

[8] Ooi, L.-G. and Liong, M.-T. (2010) Cholesterol-Lowering Effects of Probiotics and Prebiotics: A Review of in Vivo and in Vitro Findings. International Journal of Molecular Science, 11, 2499-2522. http://dx.doi.org/10.3390/ijms11062499

[9] Pereira, D.I.A. and Gibson, G.R. (2002) Effects of Consumption of Probiotics and Prebiotics on Serum Lipid Levels in Humans. Critical Reviews Biochemistry Molecular Biology, 37, 259-281. http://dx.doi.org/10.1080/10409230290771519

[10] Ataie-Jafari, A., Larijani, B. and Majd, H.A. (2009) Cholesterol-Lowering Effect of Probiotic Yogurt in Comparison with Ordinary Yogurt in Mildly to Moderately Hypercholesterolemic Subjects. Annals Nutrition and Metabolism, 54, 22-27. http://dx.doi.org/10.1159/000203284

[11] Xiao, J.Z., Kondo, S., Takahashi, N., Miyaji, K., Oshida, K., Hiramatsu, A., Iwatsuki, K., Kokubo, S. and Hosono, A. (2003) Effects of Milk Products Fermented by Bifidobacterium longum on Blood Lipids in Rats and Healthy Adult Male Volunteers. Journal of Dairy Science, 86, 2452-2461. http://dx.doi.org/10.3168/jds.S0022-0302(03)73839-9

[12] Anderson, J.W. and Gilliland, S.E. (1999) Effect of Fermented Milk (Yogurt) Containing Lactobacillus acidophilus L1 on Serum Cholesterol in Hypercholesterolemic Humans. Journal of the American College of Nutrition, 18, 43-50. http://dx.doi.org/10.1080/07315724.1999.10718826

[13] De Roos, N.M., Schouten, G. and Katan, M.B. (1999) Yogurt Enriched with Lactobacillus acidophilus Does Not Lower Blood Lipids in Healthy Men and Women with Normal to Borderline High Serum Cholesterol Levels. European Journal of Clinical Nutrition, 53, 277-280. http://dx.doi.org/10.1038/sj.ejcn.1600722

[14] Lewis, S.J. and Burmeister, S. (2005) A Double-Blind Placebo-Controlled Study of the Effects of Lactobacillus acidophilus on Plasma Lipids. European Journal of Clinical Nutrition, 59, 776-780. http://dx.doi.org/10.1038/sj.ejcn.1602139

[15] Simons, L.A., Armansec, S.G. and Conway, P. (2006) Effect of Lactobacillus fermentum on Serum Lipids in Subjects with Elevated Serum. Nutrition, Metabolism and Cardiovascular Disease, 16, 531-535. http://dx.doi.org/10.1016/j.numecd.2005.10.009

[16] Taranto, M.P., Medici, M., Perdigon, G., Ruiz Holgado, A.P. and Valdez, G.F. (2000) Effect of Lactobacillus reuteri on the Prevention of Hypercholesterolemia in Mice. Journal of Dairy Science, 83, 401-403. http://dx.doi.org/10.3168/jds.S0022-0302(00)74895-8

[17] Taranto, M.P., Medici, M., Perdigon, G., Ruiz Holgado, A.P. and Valdez, G.F. (1998) Evidence for Hypocholesterolemic Effect of Lactobacillus reuteri in Hypercholesterolemic Mice. Journal of Dairy Science, 81, 2336-2340. http://dx.doi.org/10.3168/jds.S0022-0302(98)70123-7 
[18] International Physical Activity Questionnaire (IPAQ). (2010) https://docs.google.com/viewer?a=v\&pid=sites\&srcid=ZGVmYXVsdGRvbWFpbnx0aGVpcGFxfGd4OjJhOTZhOGF hMWNjNzIONTI

[19] Salas-Salvadó, J., Fernandez-Ballart, J., Ros, E., Martinez-Gonzalez, N.A. et al., PREDIMED Study Investigators. (2008) Effect of the Mediterranean Diet Supplemented with Nuts on Metabolic Syndrome Status. Archives of Internal Medicine, 168, 2449-2458. http://dx.doi.org/10.1001/archinte.168.22.2449

[20] World Health Organization (2001) STEPwise Approach to Surveillance (STEPS). http://www.who.int/chp/steps/Instrumento_STEPS_v2.1_ES.pdf

[21] Agerbaek, M., Gerdes, L.U. and Richelsen, B. (1995) Hypocholesterolaemic Effect of a New Fermented Milk Product in Healthy Middle-Aged Men. European Journal of Clinical Nutrition, 49, 346-352.

[22] Agerholm-Larsen, L., Raben, A., Haulrik, N., Hansen, A.S., Manders, M. and Astrup, A. (2000) Effect of 8 Week Intake of Probiotic Milk Products on Risk Factors for Cardiovascular Diseases. European Journal of Clinical Nutrition, 54, 288-297. http://dx.doi.org/10.1038/sj.ejcn.1600937

[23] Bertolami, M.C., Faludi, A.A. and Batlouni, M. (1999) Evaluation of the Effects of a New Fermented Milk Product (Gaio) on Primary Hypercholesterolemia. European Journal of Clinical Nutrition, 53, 97-101. http://dx.doi.org/10.1038/sj.ejcn.1600683

[24] Sessions, V.A., Lovegrove, J.A., Dean, T.S., Williams, C.M., Sanders, T.A.B., Macdonald, I.A. and Salter, A.M. (1998) The Effect of a New Fermented Milk Product on Plasma Cholesterol and Apolipoprotein B Concentrations in MiddleAged Men and Women. In: Sadler, M.J. and Saltmarch, M., Eds., Functional Foods-The Consumer, the Products and the Evidence, Royal Society of Chemistry, London, 15-19.

[25] Schaafsma, G., Meuling, W.J., Van Dokkum, W. and Bouley, C. (1998) Effects of a Milk Product, Fermented by Lactobacillus acidophilus and with Fructo-Oligosaccharides Added, on Blood Lipids in Male Volunteers. European Journal of Clinical Nutrition, 52, 436-440. http://dx.doi.org/10.1038/sj.ejcn.1600583

[26] Lin, S.Y., Ayres, J.W., Winkler Jr., W. and Sandine, W.E. (1989) Lactobacillus Effects on Cholesterol: In Vitro and in Vivo Results. Journal of Dairy Science, 72, 2885-2899. http://dx.doi.org/10.3168/jds.S0022-0302(89)79439-X

[27] Sadrzadeh-Yeganeh, H., Elmadfa, I., Djazayery, A., Jalali, M., Heshmat, R. and Chamary, M. (2010) The Effects of Probiotic and Conventional Yoghurt on Lipid Profile in Women. British Journal of Nutrition, 103, 1778-1783. http://dx.doi.org/10.1017/S0007114509993801

[28] Jones, M.L., Martoni, C.J., Parent, M. and Prakash, S. (2012) Cholesterol-Lowering Efficacy of a Microencapsulated Bile Salt Hydrolase-Active Lactobacillus reuteri NCIMB 30242 Yoghurt Formulation in Hypercholesterolaemic Adults. British Journal of Nutrition, 107, 1505-1513. http://dx.doi.org/10.1017/S0007114511004703

[29] Jones, M.L., Martoni, C.J. and Prakash, S. (2012) Cholesterol Lowering and Inhibition of Sterol Absorption by Lactobacillus reuteri NCIMB 30242: A Randomized Controlled Trial. European Journal of Clinical Nutrition, 66, 12341241. http://dx.doi.org/10.1038/ejcn.2012.126

[30] Guo, Z., Liu, X.M., Zhang, Q.X., Shen, Z., Tian, F.W., Zhang, H., Sun, Z.H., Zhang, H.P. and Chen, W. (2011) Influence of Consumption of Probiotics on the Plasma Lipid Profile: A Meta-Analysis of Randomized Controlled Trials. Nutrition, Metabolism and Cardiovascular Disease, 21, 844-850. http://dx.doi.org/10.1016/j.numecd.2011.04.008

[31] Manson, J.E., Tosteson, H., Satterfield, S., Hebert, P., O’Connor, G.T., Buring, J.E. and Hennekens, C.H. (1992) The Primary Prevention of Myocardial Infarction. New England Journal of Medicine, 326, 1406-1416. http://dx.doi.org/10.1056/NEJM199205213262107 